\title{
Perfil da aids em indivíduos acima de 50 anos na região amazônica
}

\author{
Aids profile in individuals over 50 years old in the Amazon region
}

Gabriel de Deus Vieiral

Thaianne da Cunha Alves'

Camila Maciel de Sousa ${ }^{1}$

\section{Resumo}

O número de indivíduos acima de 50 anos no Brasil vem aumentando nos últimos anos, juntamente com a quantidade de casos de aids que atinge esta população. Esses indivíduos sofrem vários problemas sociais de familiares e dos profissionais da saúde após o diagnóstico da doença, pois estes ainda consideram o idoso um ser assexuado. Assim, o presente estudo teve como objetivo estudar os dados de aids em indivíduos com 50 anos ou mais no município de Porto Velho-RO. Os dados foram coletados na AGEVISA/RO com base nos dados epidemiológicos dos sistemas de informação de agravo de notificação Sinan NET e SinanW. As variáveis analisadas foram: faixa etária, gênero, modo de transmissão, ano de diagnóstico e evolução do caso. Foram notificados 1.668 casos de aids na faixa etária entre 14 e 81 anos, dos quais 15\% (251) correspondiam a indivíduos acima de 50 anos, sendo 69,4\% (175) homens e 30,4\% (76) mulheres. O modo de transmissão do vírus mais comum foi relação sexual exclusiva com mulheres (144 casos) e o modo de transmissão com menor número de casos foi por transfusão sanguínea, com um caso. Na evolução dos casos, 191 pacientes estavam em tratamento e 54 foram a óbito. Houve aumento do número de indivíduos acima de 50 anos com aids na última década no município de Porto Velho-RO.

\section{Abstract}

The number of individuals over 50 years in Brazil has increased in recent years along with the number of AIDS cases in this population. These individuals suffer from various social problems with family and health professionals after diagnosis, because these still consider the elder an asexual being. Thus, this study aimed to assess data on AIDS in individuals aged 50 or older in the city of Porto Velho, state of Rondonia, Brazil. Data were collected in AGEVISA / RO based on epidemiological data of information systems Sinan NET and SinanW. The variables analyzed were: age, gender, mode of transmission, year of diagnosis and outcome. Were reported 1,668 AIDS cases in the age group between 14 and 81 years, of which 15\% (251) were individuals over 50 years, and $69.4 \%$ (175) were men and $30.4 \%$ (76) women. The most common mode of

Palavras-chave:

Epidemiologia. Síndrome de Imunodeficiência Adquirida. Idoso.
Key words: Epidemiology. Acquired Immunodeficiency Syndrome. Elderly.

\footnotetext{
Departamento de Medicina. Faculdade São Lucas. Porto Velho, RO, Brasil.
} 
transmission of the virus was exclusive sexual relationship with women (144 cases) and transmission mode with a smaller number of cases by blood transfusion was having an affair. In the course of the disease, 191 patients were treated and 54 died. There was an increase in the number of individuals older than 50 years with AIDS in the last decade in the city of Porto Velho-RO.

\section{INTRODUÇÃo}

Nos últimos anos, tem-se observado um aumento no número de casos da síndrome da imunodeficiência adquirida (Aids), tanto em mulheres quanto em homens com idade superior ou igual a 50 anos, o que demonstra ser o indício de uma nova característica da doença. ${ }^{1}$

A relação sexual, por uma questão de senso comum, é considerada uma atividade mais frequente na população jovem; desta forma, a ideia de que indivíduos acima de 50 anos também possam manter relações sexuais não é uma realidade bem aceita na sociedade. Apesar de todos os tabus sociais, pessoas acima de 50 anos ainda possuem o desejo sexual, não tendo motivo para que o prazer e o ato sexual sejam inibidos. $^{2}$

Feitoza et al. ${ }^{3}$ mostram que a aids na população idosa apresenta grande importância epidemiológica, não pelos números absolutos, mas pelas taxas de incidência (7,6 casos para 100.000 habitantes), prevalência (224,9 para 100.000 habitantes no sexo masculino), letalidade $(43,9 \%)$ e anos potenciais de vida perdidos, que chegam a até 15 anos. ${ }^{1}$

Um fato preocupante é que, em grande parte dos casos, só se descobre a aids no idoso quando esta já se encontra em estágios avançados, o que torna difícil o tratamento com antivirais e diminui a expectativa de vida. O diagnóstico dessa síndrome nas pessoas idosas é mais difícil de realizar, porque nesta faixa etária são muito comuns doenças oportunistas e, consequentemente, os sintomas da aids podem ser ignorados. Outro fator é que grande parte dos profissionais de saúde não acredita que a doença possa atingir esses indivíduos. ${ }^{4,5}$
O aumento do número de indivíduos acima de 50 anos com aids no Brasil e no mundo é um fato atual, que pode ser detectado, avaliado e discutido com base em dados demográficos, analisando o aumento do número de notificações e o envelhecimento da população com a doença. ${ }^{6,7}$ Devido à elevação de casos de indivíduos com aids nesta faixa etária, o presente estudo teve como objetivo analisar os dados da aids em indivíduos com 50 anos ou mais no município de Porto Velho-RO.

\section{METODOLOGIA}

Trata-se de estudo epidemiológico descritivo de casos de aids na população acima de 50 anos de idade do município de Porto Velho-RO. Foram analisados os dados disponibilizados pela Agência de Vigilância Sanitária do Estado de Rondônia (AGEVISA/RO), com base nas fichas de notificação compulsória da aids, por meio dos sistemas de informação de agravo de notificação Sinan NET e SinanW. Os dados coletados foram referentes ao período de janeiro de 2000 a agosto de 2011. As variáveis estudadas foram: faixa etária, gênero, modo de transmissão, ano de diagnóstico e evolução do caso.

\section{RESULTADOS}

Verificou-se que a incidência da aids em indivíduos com 50 anos ou mais vem aumentando progressivamente no município de Porto VelhoRO. Durante esse período foram notificados 1.668 casos na faixa etária de 14 a 81 anos, dos quais 15\% (251) correspondiam a indivíduos com 50 anos ou mais. Desse total, 69,4\% (175) eram homens e $30,4 \%$ (76) mulheres.

Observando a taxa de casos em ambos os sexos na população idosa, notou-se uma inconstância 
no número de casos na população feminina; embora os casos sejam sempre menores que a taxa masculina, os casos notificados variaram bastante. Isto pode ser verificado ao se analisar os anos de 2008, 2009 e 2010, já que em 2008, dos 18 casos, três correspondiam a mulheres (figura 1). No ano seguinte, 2009, foram diagnosticados 20 casos de mulheres soropositivas, sendo que naquele ano foram notificados 48 casos em ambos os sexos. No ano de 2010 verificou-se que o número de casos diminuiu em relação a 2009, uma vez que dos 25 casos notificados, nove referiam-se a mulheres.

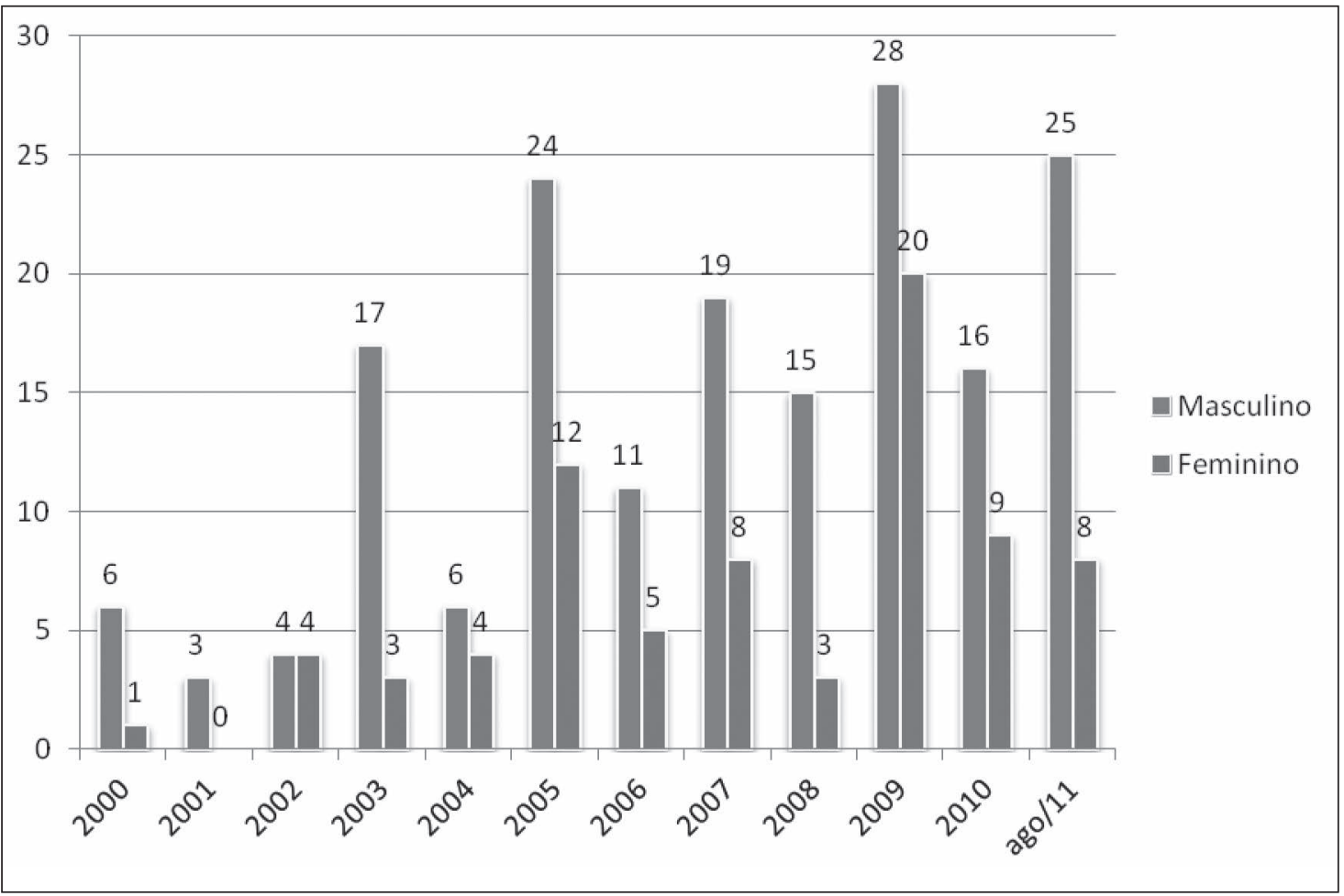

Figura 1. Indivíduos acima de 50 anos de idade com Aids, por gênero e ano de diagnóstico. Porto Velho-RO, 2000-2011.

Fonte: SINANW e SINAN NET.

O tipo de transmissão do vírus da imunodeficiência humana (HIV) varia, mas o mais comum é por relações sexuais, sendo que 236 pessoas se infectaram por essa via. Outro fato notado foi que o número de indivíduos idosos contaminados pelo HIV por meio de relações sexuais aumentou no decorrer dos anos, sendo que entre 2000 e 2006 foram notificadas 45 pessoas e entre 2007 e agosto de 2011, 191.

De acordo com a figura 2, o modo de transmissão sexual que apresentou maior número de casos de contaminação pelo HIV foi a relação sexual exclusiva com mulheres (144 casos), correspondendo a 57\%. Já a relação sexual exclusiva com homens foram 84 casos (33\%) e a relação sexual com homens e mulheres, oito (3\%); nove casos $(3,8 \%)$ foram ignorados devido ao não preenchimento desse dado na ficha de notificação.

As menores incidências de transmissão pelo HIV foram drogas injetáveis e por transfusão sanguínea. Quanto às drogas injetáveis, foram relatados quatro casos; a contaminação por transfusão sanguínea mostrou dois casos durante o período analisado. Não foi notificado nenhum caso por acidente biológico (figura 2). 


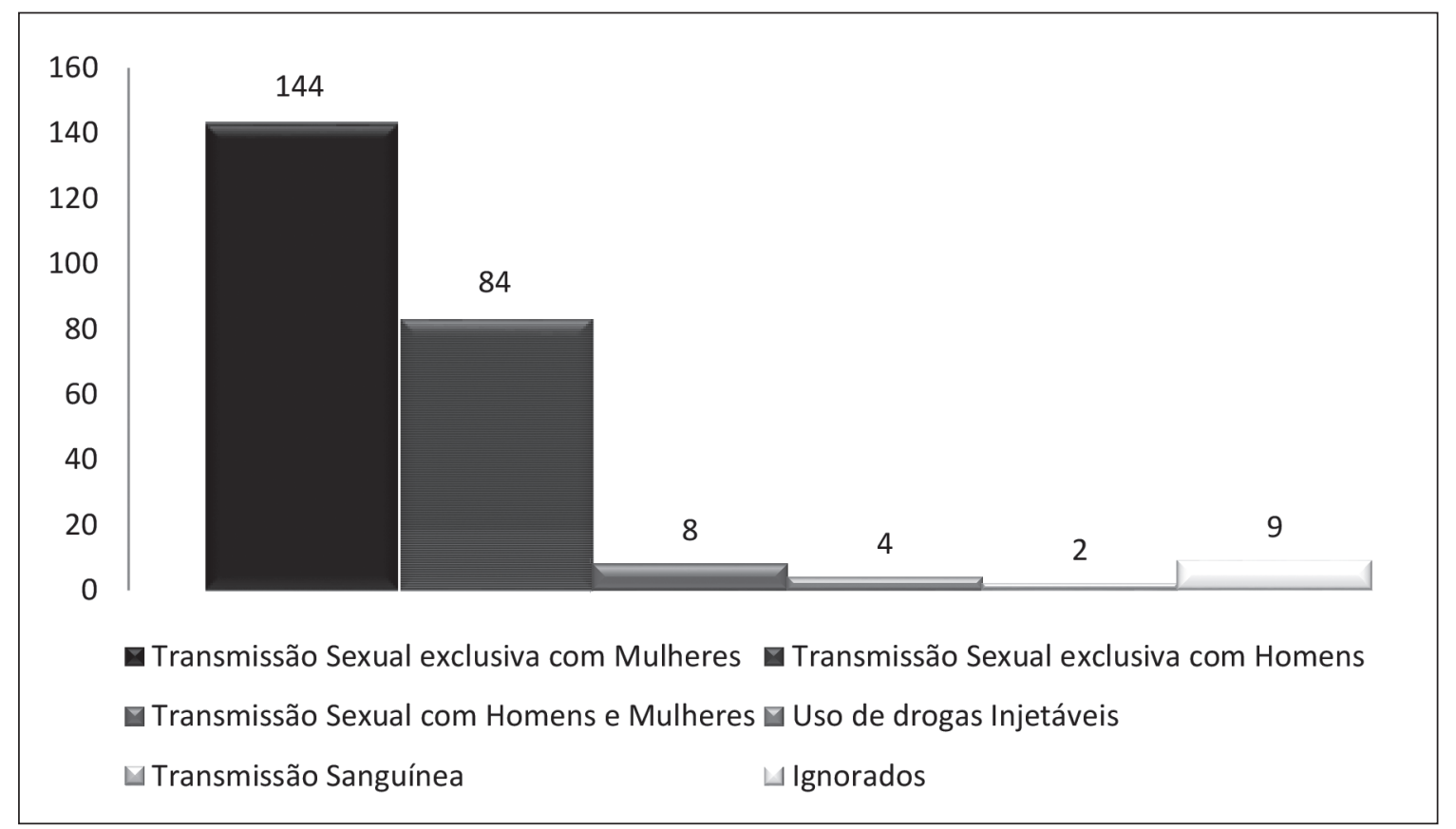

Figura 2. Percentual de transmissão do HIV em indivíduos acima de 50 anos de idade. Porto VelhoRO, 2000-2011.

Fonte: SINANW e SINAN NET.

Dos 251 idosos analisados, $21 \%$ vieram a óbito devido a aids ou a algum agravo associado à imunodeficiência, e $76 \%$ dos indivíduos se encontravam vivos; $2,4 \%$ foram considerados como ignorados, não se sabendo a evolução dos casos devido à falta de dados.

\section{DISCUSSÃO}

A qualidade dos dados epidemiológicos é um dos fatores que limitam o uso desses dados na análise da situação de saúde na população como um todo, havendo grande número de informações ignoradas. ${ }^{7}$ Neste estudo, algumas variáveis analisadas obtiveram casos ignorados, como no tipo de transmissão do vírus $(3,8 \%)$ e na evolução do caso $(2,4 \%)$.

O número de pessoas acima de 50 anos com HIV/Aids no Brasil vem aumentando significativamente: no período de 1980 a 2006, foram notificados 38.267 casos. Desse total, 26.014 eram do sexo masculino e 12.253, do feminino. Já no ano de 2006, foram registrados 976 novos casos de aids entre indivíduos do sexo masculino e 656 entre as mulheres. ${ }^{8}$

Os avanços da medicina e da indústria farmacêutica tornaram as pessoas da terceira idade mais vulneráveis a doenças sexualmente transmissíveis, como a aids, pelo fato de a vida sexual dos idosos ter sido prolongada com medicamentos que auxiliam a ereção, e também devido ao prolongamento do prazer nas idosas com o uso da terapia de reposição hormonal, que contribui para uma vida sexual mais contínua e equilibrada. ${ }^{9}$

De acordo com a figura 1, o aumento do número de casos passou a ser notado a partir de 2003, quando foram notificados 20 casos contra sete em 2000, sendo um do gênero feminino e seis do masculino. A partir daí os dados passaram a variar, tendo seu pico em 2009, quando foram registrados 48 casos, sendo 20 na população feminina e 28 na masculina. Isso demonstra que nos últimos anos vem ocorrendo aumento da 
população idosa feminina com aids, o que gerou uma série de discussões no Brasil e no mundo, com a tendência de "feminização" da epidemia."

Oestudo sobreaincidência daaids na população acima de 50 anos no município de Porto VelhoRO também demonstra aumento da população feminina quando se comparam os anos de 2001 e 2009, evidenciando aumento significativo do número de casos. No ano de 2001, não foi notificado nenhum caso de aids na população idosa feminina de Porto Velho-RO; já em 2009, foram notificados 20 casos. Isso é demonstrado não somente nos dados epidemiológicos da aids em Porto Velho, mas em outras regiões do Brasil, como no estudo realizado por Ribeiro \& Jesus, ${ }^{10}$ no Estado de Minas Gerais, onde constataram diminuição acentuada da diferença entre os sexos afetados pela doença, evidenciando o aumento do número de casos de aids na população feminina acima dos 50 anos.

Sousa et al. ${ }^{6}$ notaram que o alastramento da aids no Brasil vem gerando certas peculiaridades, apresentando mudanças em seu desenvolvimento e distribuição geográfica. Segundo apontam, inicialmente a aids era considerada uma doença restrita de jovens e indivíduos que se enquadravam em grupos de riscos, tais como os homossexuais e usuários de drogas injetáveis. Esse preconceito, porém, acabou diminuindo, pelo fato de ter sido comprovado que a aids pode afetar qualquer indivíduo da sociedade, independentemente de orientação sexual, credo, raça, condição financeira, gênero ou idade.

Pode-se observar, nos resultados obtidos neste estudo, que nos últimos dez anos a taxa de incidência de indivíduos infectados com 50 anos de idade ou mais foi de $15 \%$, atingindo tanto homens quanto mulheres, e tanto heterossexuais quanto homossexuais.

Segundo Monteiro, ${ }^{11}$ analisadas todas as faixas etárias, os heterossexuais são a população-chave sob maior risco, seguidos por homossexuais e bissexuais. Neste estudo foram observados os mesmos resultados obtidos por Monteiro, sendo a transmissão sexual entre heterossexuais maior do que a transmissão por homossexuais e bissexuais.
Gross, ${ }^{12}$ em sua dissertação, demonstrou que $82 \%$ dos idosos não usam preservativos diariamente, alegando dificuldade para se adaptarem, por sofrerem preconceitos da sociedade e simplesmente pelo sentimento de vergonha que possuem ao comprar ou conseguir seus próprios preservativos. Isso faz com que os idosos se tornem um grupo de maior risco para doenças sexualmente transmissíveis (DSTs).

Em pesquisa com idosos, Rissardo et al. ${ }^{13}$ também verificaram a tendência de não usarem preservativos durante o ato sexual. Quando indagados sobre como era feita a prevenção de DSTs, todos responderam que era com o uso de preservativos, mas mesmo sabendo disso, a maioria não usava preservativo, pois pensava ser imune às DSTs, por terem confiança no parceiro ou pelo incômodo de colocar.

Contudo, mesmo sendo diagnosticados com aids, os idosos possuem alta taxa de sobrevida, fato que vem ocorrendo a partir de 1996, devido ao desenvolvimento da terapia antirretroviral. Essa terapia aumentou a eficiência no prognóstico e aumentou a expectativa e a qualidade de vida dos pacientes com aids, mas fatores como a possível resistência do vírus à terapia e a toxicidade dos medicamentos acabam dificultando o tratamento. ${ }^{3,4}$ Os medicamentos devem ser prescritos de forma individualizada, pois cada organismo responde de maneira diferente aos fármacos, facilitando assim a adesão desses indivíduos ao tratamento. ${ }^{14}$

Programas de prevenção e educação em saúde devem ser voltados a indivíduos acima de 50 anos, com o intuito de informar e alertar a população idosa sobre a aids, discutindo temas relacionados à sexualidade na terceira idade, visando quebrar tabus e discutir as repercussões do preconceito. ${ }^{7,13,15}$

Neste estudo, algumas limitações foram encontradas e, posteriormente, servem como instrumento de pesquisa para outros autores, como uma análise detalhada dos fatores psicossociais que acometem esses indivíduos após o diagnóstico da aids, o grau de adesão à terapia antirretroviral e o tipo de relação sexual 
implicado na transmissão do HIV - se é do tipo vaginal, anal, oral, insertiva ou receptiva.

\section{CONCLUSÃO}

$\mathrm{Na}$ análise dos dados epidemiológicos da aids, evidenciou-se aumento do número de casos da doença em indivíduos acima de 50 anos de idade em Porto Velho-RO, atingindo 15\% do número total dos casos registrados no município, no período de janeiro de 2000 a agosto de 2011. Constatou-se que o principal meio de transmissão do HIV, na amostra pesquisada, se deu por

\section{REFERÊNCIAS}

1. Saldanha AAW, Araújo LF. A Aids na terceira idade na perspectiva dos idosos, cuidadores e profissionais de saúde. In: VII Congresso Virtual HIV/Aids: o VIH/SIDA na criança e no idoso; 2006.

2. Brasil. Ministério da Saúde, Secretaria de Atenção à Saúde, Departamento de Atenção Básica. Envelhecimento e saúde da pessoa idosa. Brasília: Ministério da Saúde; 2007. 192 p. (Série A. Normas e manuais técnicos ); (cadernos de atenção básica; 19).

3. Feitoza RA, Sousa AR, Araújo MFM. A magnitude da infecção pelo HIV- Aids em maiores de 50 anos no Município de Fortaleza-CE. DST J Bras Doenças Sex Transm 2004;16(4):32-7.

4. Pereira GS, Borges CI. Conhecimento sobre HIV/ Aids de participantes de um grupo de idosos, em Anápolis-Goiás. Esc Anna Nery 2010:14(4):720-5.

5. Kramer AS, Lazzarotto AR, Sprinz E, Manfroi WC. Alterações metabólicas, terapia antirretroviral e doença cardiovascular em idosos portadores de HiV. Arq Bras Cardiol 2009;93(5):561-8.

6. Sousa ACA, Suassuna DSB, Costa SML. Perfil clínico-epidemiológico de idosos de Aids. DST J Bras Doenças Sex Transm 2009;21(1):22-6.

7. Pottes FA, Brito AM, Gouveia GC, Araújo EC, Carneiro RM. Aids e envelhecimento: características dos casos com idade igual ou maior que 50 anos em Pernambuco, de 1990 a 2000. Rev Bras Epidemiol 2007;10(3):338-51

8. Boletim Epidemiológico Aids e DST. Brasília, DF: Ministério da Saúde. Ano 3, nº 1, 2006 - . relações sexuais e que houve predomínio de indivíduos do gênero masculino com a doença.

Acredita-se que o número de indivíduos soropositivos seja maior do que o apresentado neste estudo, pois foram analisados somente indivíduos com aids, sendo que a pessoa que possui a infecção pelo HIV não necessariamente irá desenvolver aids. Este é um motivo para que as autoridades públicas de saúde continuem investindo em políticas de prevenção voltadas à sexualidade, tanto na população jovem quanto na idosa.

9. Lazzarotto AR, Kramer AS, Hadrich M, Tonin MM, Caputo PMS, Sprinz E. O conhecimento de HIV/aids na terceira idade: estudo epidemiológico no Vale do Sinos, Rio Grande do Sul, Brasil. Ciênc saúde coletiva 2008; 13(6):1833-40.

10. Ribeiro LCC, Jesus MVN. Avaliando a incidência dos casos notificados de AIDS em idosos no Estado de Minas Gerais no período de 1999 a 2004. Cogitare Enferm 2006;11(2):113-6.

11. Monteiro JP. Análise da variabilidade genética do vírus da imunodeficiência humana (HIV): epidemiologia molecular no estado da Bahia [Tese]. Salvador: Fundação Oswaldo Cruz; 2009.

12. Gross JB. Estudo de pacientes portadores de HIV/ AIDS após os 60 anos de idade em duas Unidades de Saúde do Estado do Rio de Janeiro [Dissertação]. Rio de Janeiro: Fundação Oswaldo Cruz; 2005.

13. Rissardo LK, Furlan MCR, Aguiar JE. Sexualidade na terceira idade: Nível de conhecimento dos idosos em relação as DST’s. In: Simpósio Internacional de Educação Sexual da UEM; abril 2009; p.76-77. Maringá, PR: UEM; 2009.

14. Bonolo PF, Gomes RRFM, Guimarães MDC. Adesão à terapia anti-retroviral (HIV/aids): fatores associados e medidas da adesão. Epidemiol Serv Saúde 2007;16(4):267-78.

15. Brasileiro M, Freitas MIF. Representações sociais sobre a AIDS de pessoas acima de 50 anos de idade, infectadas pelo HIV. Rev Latino-am Enfermagem 2006;14(5):1-8. 